\title{
Melatonin to Reduce Death Toll Due to COVID-19: From Innate to Adaptive Immune Response
}

By Jan Tesarik

Abstract- This paper highlights a new, nonspecific medication, which could be used both as a preventive and a curative measure to slow down the progression of Coronavirus disease 2019 (COVID-19) until a more specific treatment is available. The suggested treatment (immunomodulation) consists in the administration of melatonin, a substance shown to inhibit the innate (blind and usually harmful) immune response while facilitating the adaptive one, the only capable of fighting efficiently against the infection. In low oral doses, melatonin can be administered preventively to persons at risk and those already infected but still asymptomatic. High, intravenously administered doses may help critical patients under imminent threat of death. The combined use of both strategies will hopefully unblock the current overcharge of intensive care units by reducing new admissions and favoring healed patient discharge.

Keywords: melatonin, coronavirus, SARS-CoV-2, COVID-19 prevention, COVID-19 treatment, immunomodulation, innate immune response, adaptive immune response, unblocking ICUs.

GJMR-K Classification: NLMC Code: QW 640

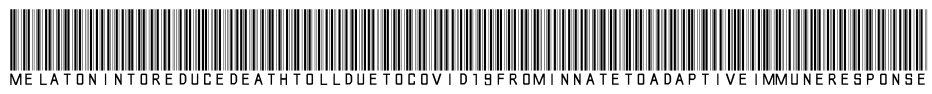

Strictly as per the compliance and regulations of:

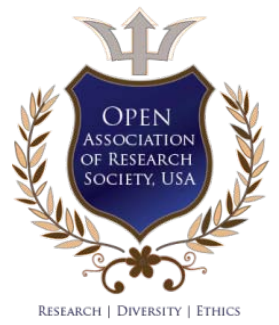

(c) 2020. Jan Tesarik. This is a research/review paper, distributed under the terms of the Creative Commons AttributionNoncommercial 3.0 Unported License http://creativecommons.org/licenses/by-nc/3.0/), permitting all non-commercial use, distribution, and reproduction in any medium, provided the original work is properly cited. 


\title{
Melatonin to Reduce Death Toll Due to COVID-19: From Innate to Adaptive Immune Response
}

\author{
Jan Tesarik
}

Abstract-This paper highlights a new, nonspecific medication, which could be used both as a preventive and a curative measure to slow down the progression of Coronavirus disease 2019 (COVID-19) until a more specific treatment is available. The suggested treatment (immunomodulation) consists in the administration of melatonin, a substance shown to inhibit the innate (blind and usually harmful) immune response while facilitating the adaptive one, the only capable of fighting efficiently against the infection. In low oral doses, melatonin can be administered preventively to persons at risk and those already infected but still asymptomatic. High, intravenously administered doses may help critical patients under imminent threat of death. The combined use of both strategies will hopefully unblock the current overcharge of intensive care units by reducing new admissions and favoring healed patient discharge.

Keywords: melatonin, coronavirus, SARS-CoV-2, COVID19 prevention, COVID-19 treatment, immunomodulation, innate immune response, adaptive immune response, unblocking ICUs.

\section{INTRODUCTION}

$\mathrm{V}$ /iruses of the Coronaviridae family are endemic in the human populations, responsible for $15-30 \%$ of respiratory tract infections each year. They usually cause common respiratory infections without lifethreatening complications. ${ }^{1}$ Due to presumably spontaneous mutations, some viruses of the Coronaviridae family became resistant to the human immune defense and caused major, more or less geographically restricted outbreaks. Severe Acute Respiratory Syndrome Coronavirus (SARS-CoV), between 2002 and 2003, followed by that of Middle Eastern Respiratory Syndrome Coronavirus (MERSCoV), between 2012 and 2015, were both due to a mutated virus of the Coronaviridae family. ${ }^{1}$ The current pandemic of Coronavirus disease 2019 (COVID-19), caused by a mutated Coronavirus of the same family, named SARS-CoV-2, is a kind of "déjà vu" in this respect. However, the rapid expansion of the contangion across the world, along with the high mortality in some populations, has brought COVID-19 into the focus of the current health concerns.

Corresponding Author: MD, PhD, MARGen Clinic, Granada, Spain. e-mail: jtesarik@clinicamargen.com
II. What is KNOWN AND What CAN BE EXPECTED

The complete genome sequence of SARS-CoV2 is now available and shares $79.0 \%$ nucleotide identity to SARS-CoV, and $51.8 \%$ identity to MERS-COV, ${ }^{2}$ indicating a high genetic homology among SARS-Cov-2, MERS-CoV and SARS-CoV-2. ${ }^{1}$ From here ahead, an efficient vaccine might be available in a relatively short time. However, this time cannot be calculated with accuracy, and it is precious because people are dying, and the emergency services of many countries lack an adequate preparation for this unexpected situation. Intensive care units (ICUs) in most countries hit by COVID-19 pandemic suffered, or are still suffering, from problems of human resources as well as a shortage of material and equipment required for facing the current situation with adequate efficiency and safety. Hence, while waiting for the development of a specific treatment for COVID-19, all that we need is time.

\section{iil. How to Gain Precious Time}

Despite the supposedly promising preliminary results with a range of antiviral drugs, including the antimalarials chloroquine and hydroxychloroquine, the antiretrovirals lopinavir/ritonavir and other antivirals, such as oseltamivir, umifenovir, remdesivir and favipiravir, the global conclusion is that no proven effective therapies for SARS-Cov-2 currently exist. ${ }^{3}$ There are two ways of gaining time required to alleviate the increasing pressure for ICUs. First, by the administration of highly efficient, though nonspecific treatments enabling the attenuation of COVID-19 symptoms to allow patients to leave ICUs as early as possible. Second, by using nonspecific preventive or disease-attenuating treatments in healthy persons at risk of contagion and in those already infected but still asymptomatic to reduce the entry of new patients into ICUs. Melatonin is not likely to be viricidal, but it has indirect protective actions against infection due to its anti-inflammatory, antioxidant, and immunomodulatory features (reviewed in Zhang et al. ${ }^{1}$ ). The data available today, though obtained with relatively limited patient populations, suggest a reduction of lifethreatening complications of different respiratory distress conditions, both those caused by viral infections, such as SARS, ${ }^{4}$ MERS, ${ }^{4}$ and Ebola ${ }^{5}$ viruses, and those caused by other etiological factors, such as newborn asphyxia, ${ }^{6}$ by intravenous administration of high doses of melatonin (Table 1). 
Table 1: Summary of the main data supporting the potential of melatonin to attenuate symptoms of severe respiratory distress

\begin{tabular}{lccc}
\hline Disease & Years & Reported effects & Reference \\
\hline $\begin{array}{l}\text { SARS } \\
\text { immune response }\end{array}$ & $2002-2003$ & Shift from innate to adaptive & Tan and Hardeland ${ }^{4}$ \\
$\begin{array}{l}\text { MERS } \\
\text { immune response }\end{array}$ & $2012-2015$ & Shift from innate to adaptive & Tan and Hardeland ${ }^{4}$ \\
$\begin{array}{l}\text { Ebola } \\
\text { shock syndrome } \\
\text { Newborn asphyxia }\end{array}$ & $2014-2016$ & Attenuation of hemorrhagic & Reiter et al. ${ }^{5}$ \\
& $2015-2016$ & $\begin{array}{l}\text { Improvement of long-term } \\
\text { neurodevelopment }\end{array}$ & Jerez-Calero et al. ${ }^{6}$ \\
\hline
\end{tabular}

The other way to act is to prevent or minimize the infection by SARS-CoV-2 with the use of relatively low daily doses of melatonin (5-10 mg administered orally). This strategy is supposed to avoid COVID-19 contagion and, if it has already occurred, to slow down the disease propagation until a specific treatment is available. In this way, ICUs will not be overwhelmed with an unexpected load of critical patients and will be able to manage patients with better selectivity and specificity, according to the particular condition of each of them.

Most of the deadly effects of COVID-19 infection are not caused directly by the virus, but rather by an inadequate immune response of the infected person. All the three genetically related coronaviruses, SARS-CoV, MERS-CoV, and SARS-CoV-2, cause a similar type of reaction in the infected organism: a repressed specific (adaptative) immune response, with hypo-albuminemia, lymphopenia, neutropenia and decreased percentage of CD8+ $T$ cells. This reaction is accompanied by activation of the innate (nonspecific) immune response, leading to a marked increase in pro-inflammatory cytokines whose accumulation, referred to as "cytokine storm" eventually leads to apoptosis of epithelial and endothelial cells, vascular leakage, and abnormal T-cell and macrophage responses, which can cause the potentially life-threatening acute respiratory distress syndrome. ${ }^{1}$ This pathogenetic mechanism is not unique to viral respiratory diseases. It plays an essential role in several other human pathologies involving a hyperactivation of the innate immune response, such as the neonatal hypoxic-ischemic encephalopathy. ${ }^{6}$ It is just in this latter pathology where a recent randomized controlled trial has shown a significant improvement of long-term neurodevelopmental outcomes of the affected children with the use of high doses of intravenously administered melatonin (Table 1). ${ }^{6}$ The excellent tolerance and the lack of detectable side effects of melatonin are other arguments in favor of its preventive and curative use against COVID-19.

As compared to melatonin, most of the the recently tested antiviral drugs ${ }^{3}$ show considerable toxicity, especially in patients suffering from other pathological conditions. The same applies to the use of corticosteroids, such as dexamethasone, which is currently going "viral". The use of corticosteroids in critical COVID-19 patients was first suggested by Mehta et al. $^{7}$ to moderate the imminent risk of cytokinemediated hyper-inflammation detectable with the use of appropriate blood tests. However, a later study criticized this suggestion, pointing out that it would be hardly possible to make out the cause-effect relationship between this condition and the overall disease progression from a simple association between the two phenomena. $^{8}$ If the hyper-inflammation is a consequence of the host's failing defense against the infectious agent, rather than its cause, further weakening of the patient's immune response may have potentially fatal consequences. ${ }^{8}$ Hence, it sounds reasonable to use melatonin-mediated immunomodulation rather than corticosteroid-mediated immunosuppression, even in critical COVID-19 patients.

\section{Is IT ETHICAL TO WAit FOR RCT OutComes before We START using Melatonin Against Covid-19?}

For several decades there has been a consensus among physicians and clinical researchers that randomized controlled trials (RCTs) provide the most rigorous test to justify the application of new preventive, diagnostic and therapeutic interventions. ${ }^{9}$ However, under certain conditions, waiting for definitive conclusions from RCTs before applying a new treatment in clinical practice may be questionable for practical and ethical reasons. From the ethical point of view, RCTs should not be conducted unless there is equipoisegenuine doubt about whether one course of action is better than another, so that it is not ethical to build a trial in which evidence suggests that patients in one arm of the study are more likely to benefit from enrollment that patients in the other arm. ${ }^{10}$ This reasoning prevailed in the case of the outbreak of Ebola disease, six years ago, when $\mathrm{WHO}$ made an important statement concerning the necessity of RCTs before starting treatments. This crisis is so acute, $\mathrm{WHO}$ declared, that it is ethical to offer interventions with potential benefits but 
unknown efficacy and side effects, though every effort should be made to evaluate benefits and risks and share all data generated. ${ }^{11}$

In my opinion, a similar approach should be adopted for the use of melatonin as a preventive and curative agent in the management of COVID-19. Concerning the above $\mathrm{WHO}$ declaration, melatonin lacks notable secondary effects, even at relatively high doses, is unexpensive and immediately available, and has several properties suggesting that it may be able to prevent the development of COVID-19 symptoms, decrease the severity of already present symptoms, and reduce the immunopathology of COVID-19 after the active phase of the infection is over, with particular regard to pulmonary fibrosis. ${ }^{12}$ Adhering to the "First Do Not Harm" principle, it also has to be stressed that not only is melatonin harmless to human health, but it is beneficial, regardless of its highly probable, though not definitively proven, anti-COVID-19 action, namely as a potent antioxidant and modulator of cell signaling pathways controlling the development of different types of tumors, such as breast cancer, prostate cancer, gastric cancer, and colorectal cancer. ${ }^{13}$

\section{Conclusions}

Given the above considerations, the treatment of critical patients by intravenous administration of highdose melatonin (up to $100 \mathrm{mg}$ daily) may not only save their lives but also accelerate their discharge from ICUs, thus alleviating the current ICU overcharge. In cases of milder or asymptomatic COVID-19 infections, or as a preventive measure in persons at risk, oral administration of lower melatonin doses $(5-10 \mathrm{mg}$ ) may attenuate the evolution of the disease and thus reduce the entry of new patients into ICUs. In countries in which the COVID-19 pandemic is still on the rise, the use of melatonin as a preventive measure and therapeutic agent against COVID-19 should be taken seriously into consideration, even before the availability of RCT outcomes to confirm its benefits. In particular, it should be considered as a preventive measure to protect older adults, pregnant women, health personnel, and other professionally exposed individuals.

\section{References Références Referencias}

1. Zhang $\mathrm{R}$, Wang $\mathrm{X}, \mathrm{Ni} \mathrm{L}$, et al. Melatonin as a potential adjuvant treatment. Life Sci 2020; 250:117583. doi:10.1016/j.lfs.2020.117583.

2. Ren LL, Wang $Y M, W u Z Q$, et al. Identification of a novel coronavirus causing severe pneumonia in human: a descriptive study. Chin Med J (Engl). 2020; 133(9):1015-1024. doi: 10.1097/CM9. 0000000000000722.

3. Sanders JM, Monogue ML, Jodlowski TZ, Cutrell JB. Pharmacologic treatments for coronavirus disease
2019 (COVID-19): a review. JAMA 2020; 323(18):1824-1836. doi:10.1001/jama.2020.6019.

4. Tan D-X, Hardeland R. Potential utility of melatonin in deadly infectious diseases related to the overreaction of innate immune response and destructive inflammation: focus on COVID-19. Melatonin Res 2020; 3(1):120-143. doi: https:// doi.org/10.32794/mr11250052.

5. Reiter RJ, Ma Q, Sharma R. Treatment of Ebola and other infectious diseases: melatonin "goes viral". Melatonin Res 2020;(1)3:43-57. doi: https:// doi.org/10.32794/mr11250047.

6. Jerez-Calero A, Salvatierra Cuenca MT, Benitez Feliponi A, Fernández-Marín CE, Narbona-López E, Uberos Fernández J, Muñoz-Hoyos A. Hypothermia plus melatonin in asphyctic newborns: a randomized-controlled pilot study. Pediatr Crit Care Med. 2020; 10.1097/PCC. 0000000000002346. doi: 10.1097/PCC. 0000000000002346.

7. Mehta P, McAuley DF, Brown M, Sanchez E, Tattersall RS, Manson JJ, on behalf of the HLH Across Speciality Collaboration, UK. COVID-19: consider cytokine storm syndromes and immunosuppression. Lancet 2020; 395(10229): 1033-1034. doi: 10.1016/S0140-6736(20)30628-0.

8. Ritchie $\mathrm{Al}$, Singanayagam A. Immunosuppression for hyperinflammation in COVID-19: a double-edged sword?. Lancet 2020; 395(10230): 1111. doi: 10.1016/S0140-6736(20)30691-7.

9. Jones DS, Podolsky SH. The history and fate of the gold standard. Lancet 2015; 385(9977):1502-1503. doi:10.1016/S0140-6736(15)60742-5.

10. Duffy SW. Interpretation of the breast screening trials: a commentary on the recent paper by Gøtzsche and Olsen. Breast 2001; 10(3):209-212. doi:10.1054/brst.2000.0238.

11. Adebamowo C, Bah-Sow O, Binka F, et al. Randomised controlled trials for Ebola: practical and ethical issues. Lancet 2014; 384(9952): 14231424. doi:10.1016/S0140-6736(14)61734-7.

12. Shneider A, Kudriavtsev A, Vakhrusheva A. Can melatonin reduce the severity of COVID-19 pandemic? Int Rev Immunol 2020; 1-10. DOI: 10.1080/08830185.2020.1756284.

13. Li Y, Li S, Zhou Y, Meng X, Zhang JJ, Xu DP, Li HB. Melatonin for the prevention and treatment of cancer. Oncotarget 2017; 8(24):39896-39921. doi: 10.18632/oncotarget.16379. 